ARTICLE

DOI: $10.1038 / s 41467-017-01195-y$

\title{
DNA N6-methyladenine is dynamically regulated in the mouse brain following environmental stress
}

\author{
Bing Yao ${ }^{1}$, Ying Cheng ${ }^{1}$, Zhiqin Wang ${ }^{2}$, Yujing $\mathrm{Li}^{1}$, Li Chen ${ }^{1,5}$, Luoxiu Huang ${ }^{1}$, Wenxin Zhang ${ }^{3}$, Dahua Chen ${ }^{3}$,
} Hao $\mathrm{Wu}^{4}$, Beisha $\mathrm{Tang}^{2} \&$ Peng Jin (1) ${ }^{1}$

Chemical modifications on DNA molecules, such as 5-methylcytosine and 5-hydroxymethylcytosine, play important roles in the mammalian brain. A novel DNA adenine modification, $N(6)$-methyladenine $(6 \mathrm{~mA})$, has recently been found in mammalian cells. However, the presence and function(s) of $6 \mathrm{~mA}$ in the mammalian brain remain unclear. Here we demonstrate $6 \mathrm{~mA}$ dynamics in the mouse brain in response to environmental stress. We find that overall $6 \mathrm{~mA}$ levels are significantly elevated upon stress. Genome-wide $6 \mathrm{~mA}$ and transcriptome profiling reveal an inverse association between $6 \mathrm{~mA}$ dynamic changes and a set of upregulated neuronal genes or downregulated LINE transposon expression. Genes bearing stress-induced $6 \mathrm{~mA}$ changes significantly overlap with loci associated with neuropsychiatric disorders. These results suggest an epigenetic role for $6 \mathrm{~mA}$ in the mammalian brain as well as its potential involvement in neuropsychiatric disorders.

\footnotetext{
${ }^{1}$ Department of Human Genetics, Emory University School of Medicine, Atlanta, GA 30322, USA. ${ }^{2}$ Department of Neurology, Xiangya Hospital, Central South University, Changsha, Hunan 410008, China. ${ }^{3}$ State Key Laboratory of Reproductive Biology, Institute of Zoology, Chinese Academy of Sciences, Beijing 100101, China. ${ }^{4}$ Department of Biostatistics and Bioinformatics, Emory University School of Public Health, Atlanta, GA 30322, USA. ${ }^{5}$ Present address: Department of Health Outcomes Research and Policy, Harrison School of Pharmacy, Auburn University, Auburn, AL 36849, USA. Bing Yao and Ying Cheng contributed equally to this work. Correspondence and requests for materials should be addressed to P.J. (email: peng.jin@emory.edu)
} 
C ovalent DNA modifications on the 5-carbon position of cytosine, such as 5-methylcytosine $(5 \mathrm{mC})$ and 5hydroxymethylcytosine $(5 \mathrm{hmC})$, are well known to play critical epigenetic roles in spatially and temporally modulating neuronal gene expression in the mammalian central nervous system $(\mathrm{CNS})^{1,2}$. A novel DNA adenine modification, N6methyladenine $(6 \mathrm{~mA})$, which is prevalent in prokaryotes $^{3}$, was recently found in the genome of high eukaryotes, including green algae, worms, fruit flies, frogs, zebrafish, pigs, and mice ${ }^{4-9}$. Although evidence from these studies suggests potential epigenetic roles for $6 \mathrm{~mA}$, its precise biological function(s) remain elusive $^{10}$. Furthermore, little is known about the presence and functions of $6 \mathrm{~mA}$ in the mammalian CNS.

Various genetic factors and environmental cues contribute to mental illness ${ }^{11}$. Among these, exposure to chronic stress is one of the strongest and most direct risk factors for developing numerous psychiatric disorders including depression ${ }^{12,} 13$. Compelling evidence support the fundamental roles of epigenetic alterations in depression as changes in both DNA methylation and histone modifications alter gene expression in brains ${ }^{14}, 15$. The prefrontal cortex (PFC) is highly involved in complex cognitive behavior, but is also sensitive to the adverse effects of stress exposure $^{16}$. In rodent experiments, chronic stress results in dendritic atrophy and spine loss in the $\mathrm{PFC}^{17}$. A recent study demonstrated that chronic social defeat stress could decrease DNA methylation levels in $\mathrm{PFC}^{18}$. It remains to be determined whether the DNA adenine modification $6 \mathrm{~mA}$ is present in the mammalian brain and if it is involved in the response to extrinsic challenges such as stress.

Here we examined the dynamic changes of $6 \mathrm{~mA}$ in mouse PFC in response to stress. We found that global $6 \mathrm{~mA}$ was significantly elevated upon stress. Genome-wide $6 \mathrm{~mA}$ and transcriptome profiling revealed an inverse association of $6 \mathrm{~mA}$ dynamic changes with a set of upregulated neuronal genes or downregulated LINE transposon expression. Genes bearing stress-induced $6 \mathrm{~mA}$ changes significantly overlap with loci associated with neuropsychiatric disorders. These results together suggest a potential epigenetic role for $6 \mathrm{~mA}$ in the mammalian brain as well as its possible involvement in mental illness.

\section{Results}

Chronic stress induces the accumulation of $6 \mathrm{~mA}$ in mouse PFC. It is well known that environmental exposure may induce epigenetic changes. To understand the dynamics of $6 \mathrm{~mA}$ in the mouse brain, we employed a well-established paradigm for chronic stress to model environmental exposure. We exposed 7to 8-week-old wild-type C57BL/6 adult male mice to normal or restraint environments two hours per day in consecutive 14 days $^{19,20}$ (Supplementary Fig. 1a, b). We then performed the forced swim test (FST) and tail suspension test (TST) to evaluate stress responses in restrained and control mice. There was a significant increase in immobility time in the restrained stressed group in both the FST and TST, indicating that the restraint environment successfully altered mouse behavior (Fig. 1a, b). In order to test $6 \mathrm{~mA}$ changes in response to stress, we applied the highly sensitive ultra-performance liquid chromatography tandem mass spectrometer (UHPLC-MS/MS) to precisely quantify $6 \mathrm{~mA}$ dynamic changes upon stress in several brain regions involved in stress response, including the PFC, hippocampus (HIP), amygdala (AMY), and hypothalamus (HYP). Interestingly, although detectable $6 \mathrm{~mA}$ was present in all of these brain regions, only $6 \mathrm{~mA}$ in PFC showed substantial and significant increase from an average of 6.6 p.p.m. ( $6 \mathrm{~mA}$ per million dA) to 25.5 p.p.m. upon stress (Fig. 1c). Since PFC has been widely linked to critical neuronal activities such as stress response ${ }^{21,22}$, we validated the
$6 \mathrm{~mA}$ dynamic changes in genomic DNA isolated from control and stressed mice PFC using a 6mA-specific antibody, whose specificity to DNA $6 \mathrm{~mA}$ has been extensively validated in previous publications ${ }^{4}, 5,7,9$, and by PCR using normal dATP or 6mA-modified dATP (Fig. 1d). Consistent with UHPLC results, $6 \mathrm{~mA}$ was present in the PFC of wild-type mice and showed $\sim$ fourfold significant increase upon stress (Fig. 1d, e and Supplementary Fig. 1c). These data indicate that $6 \mathrm{~mA}$ is present in mammalian brains and undergoes dynamic changes upon stress.

Genome-wide $6 \mathrm{~mA}$ dynamics upon stress exposure. To understand the potential role of $6 \mathrm{~mA}$ in stress response, we randomly chose three pairs of control and stressed mice from three independent stress experiments to profile genome-wide $6 \mathrm{~mA}$ dynamics in PFCs using a published $6 \mathrm{~mA}$-immunoprecipitation (IP) protocol coupled with high-throughput sequencing $6,7,9$. We ensured the specificity of the $6 \mathrm{~mA}$-IP using competitive elution with excess $6 \mathrm{~mA}$-modified nucleotides. In order to identify significant differential $6 \mathrm{~mA}$ dynamic regions upon stress, we applied published computational algorithms edge $\mathrm{R}^{23}$ to compare normalized $6 \mathrm{~mA}$ read density differences between triplicated control and stressed PFC groups across the mouse genome utilizing $500 \mathrm{bp}$ bins based on the $6 \mathrm{~mA}-\mathrm{IP}$ resolution. Differential regions with a $p$-value $<0.05 \quad(-\log 10 \quad p$-value $>1.3$, false discovery rate $<0.077)$ were considered significant. We identified 21,974 loss-of-6mA regions and 37,937 gain-of-6mA regions upon stress (Fig. 2a, and Supplementary Data 1,2). Genomic annotation of these $6 \mathrm{~mA}$ dynamic regions revealed substantial biased genomic associations. For example, $65 \%$ of regions that gained $6 \mathrm{~mA}$ upon stress were associated with intergenic regions, which is the highest enrichment vs. expected value (Fig. 2b). Intragenic gain-of-6mA upon stress predominantly occurred at introns and was excluded from most coding exons ( $33 \%$-introns vs. $0.38 \%$-exons, Fig. 2 b). The strong association between $6 \mathrm{~mA}$ and introns and intergenic regions was consistent with observations in mouse kidney ${ }^{\top}$ and embryonic stem cells ${ }^{9}$. Additionally, $38 \%$ of intragenic loss-of$6 \mathrm{~mA}$ occurred at introns, among the highest enrichment vs. expected value, and $59 \%$ of loss-of- $6 \mathrm{~mA}$ were identified at intergenic regions (Fig. 2c). The fact that gain-of- $6 \mathrm{~mA}$ and lossof- $6 \mathrm{~mA}$ had differential enrichment at intergenic regions or introns vs. expected values may indicate the differential roles of $6 \mathrm{~mA}$ at these genomic regions. The $6 \mathrm{~mA}$ reads ratio (stressed vs. control) was plotted across the intragenic regions of genes bearing dynamic $6 \mathrm{~mA}$ changes upon stress, i.e., red plots (fold change $>1$ ) indicate overall gain of $6 \mathrm{~mA}$ upon stress whereas blue plots (fold change $<1$ ) indicate loss of $6 \mathrm{~mA}$ (Fig. 2d). Interestingly, more $6 \mathrm{~mA}$ loss was observed around transcription start sites (TSS) than gene bodies in genes bearing loss-of-6mA regions (Fig. $2 \mathrm{~d}$ ). In contrast, gain-of- $6 \mathrm{~mA}$ showed substantially more gene body enrichment than TSS in genes carrying gain-of- $6 \mathrm{~mA}$ regions (Fig. 2d). These observations imply that gain- or loss-of-6mA has differential roles on protein-coding genes.

6mA negatively correlates with LINE transposon expression. To further dissect the association between $6 \mathrm{~mA}$ dynamic changes upon stress and intergenic transposon activities, we analyzed intergenic $6 \mathrm{~mA}$ dynamic regions at different classes of transposon elements (Fig. 3). We found that $49.2 \%$ of intergenic gain-of- $6 \mathrm{~mA}$ was annotated to long interspersed nuclear element (LINE) transposons, which is the most highly enriched transposon over expected value (Fig. 3a). In contrast, only $8.8 \%$ of loss-of- $6 \mathrm{~mA}$ regions were located on LINEs, while $33.9 \%$ enriched at simple repeats (Fig. 3b). N(6)-methyladenine has been shown to modulate transposon expression in Drosophila ${ }^{6}$ and target LINE 
a

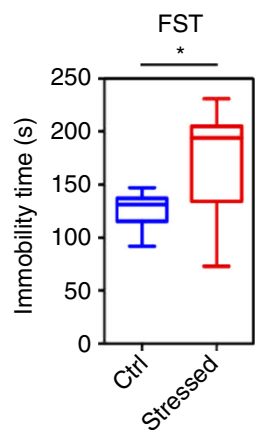

d

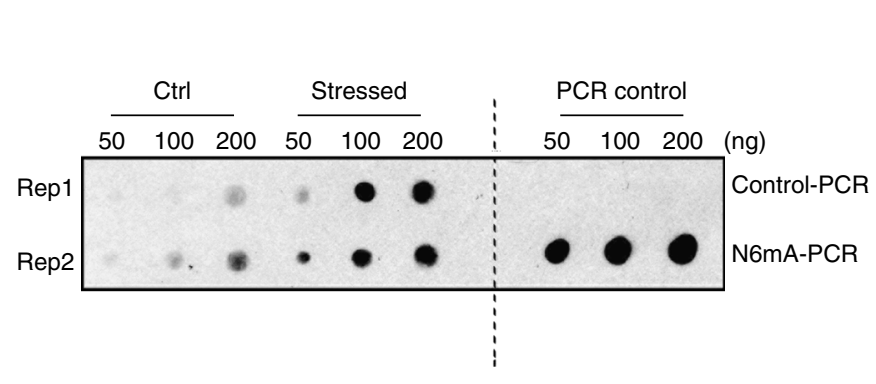

b

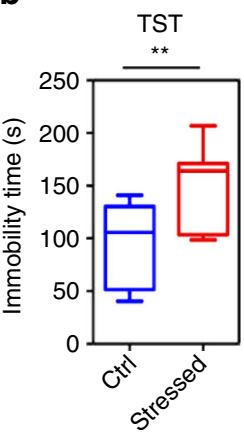

c

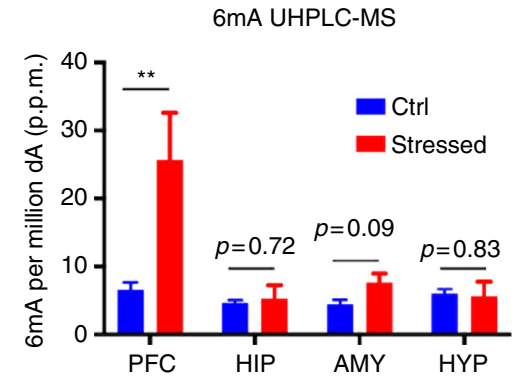

e

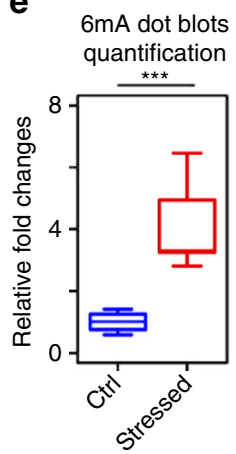

Fig. 1 Chronic stress induces $6 \mathrm{~mA}$ accumulation in mouse prefrontal cortex (PFC). $\mathbf{a}, \mathbf{b}$ Chronic stress resulted in increased immobility time in the forced swim test (FST) and tail suspension test (TST). $\left(n=9\right.$; ${ }^{*} p<0.05$; ${ }^{\star \star} p<0.01$; unpaired $t$-test, error bars $=$ mean \pm SEM, unpaired $t$-test). $\mathbf{c}$ Highly sensitive ultra-performance liquid chromatography tandem mass spectrometer (UHPLC-MS/MS) precisely quantified $6 \mathrm{~mA}$ in prefrontal cortex (PFC), Hippocampus (HIP), Amygdala (AMY), and Hypothalamus (HYP) upon stress. N(6)-methyladenine/total A was indicated as percentage per million dA (p.p.m.). N(6)methyladenine levels in PFC drastically increased from 6.6 p.p.m. ( $6 \mathrm{~mA}$ per million dA) to 25.5 p.p.m. upon stress ${ }^{{ }^{\star \star}} p<0.01$; unpaired $t$-test; nonsignificant $p$-values are indicated). d Representative $6 \mathrm{~mA}$-specific dot blots revealed accumulation of $6 \mathrm{~mA}$ in mouse PFC upon stress. Antibody specificity has been validated by detecting PCR products using methylated dATP (6mA-PCR) but not unmodified dATP (control PCR). e Quantification of dot blots in d by Image J software $\left(n=4,{ }^{\star \star \star} p<0.001\right.$; unpaired $t$-test, error bars $=$ mean \pm SEM $)$

transposons in mouse embryonic stem cell (mESC) $)^{9}$. Stress could also substantially alter transposon expression in mouse PFC (Supplementary Data 3). Expression of transposons, such as LINEs, has been proposed to play important roles in stress response in the mammalian brain ${ }^{24}$. Since $49.2 \%$ of intergenic gain-of-6mA upon stress were located on LINEs and highly enriched than expected values (Fig. 3c), which was not found in the loss-of-6mA regions (Fig. 3d), we sought to investigate gainof- $6 \mathrm{~mA}$ association with LINE expression by RNA-seq. 103 of 120 expressed LINEs in PFC had significant $6 \mathrm{~mA}$ changes upon stress, with $82(79.6 \%)$ showing increased $6 \mathrm{~mA}$. Importantly, the majority (74 out of $82,90.2 \%$ ) of LINEs with gain-of- $6 \mathrm{~mA}$ are downregulated upon stress (Fig. 3e). The percentage of downregulated LINEs bearing increased $6 \mathrm{~mA}$ was significantly more enriched than observed in short interspersed nuclear element (SINE) transposons $\left(\chi^{2}\right.$ test, $\left.p<0.001\right)$. These data indicate an inverse association between gain-of- $6 \mathrm{~mA}$ and LINE expression upon stress. This is consistent with strong enrichment of gain-of$6 \mathrm{~mA}$ annotations at LINE elements relative to expected values (Fig. 3a).

To validate the dynamic $6 \mathrm{~mA}$ regions identified by $6 \mathrm{~mA}-\mathrm{IP}$ seq, we employed a published restriction enzyme digestion method which takes advantage of the $6 \mathrm{~mA}$-sensitive restriction enzyme $D p n I$ that preferentially cleaves methylated adenine at GATC/CATC/GATG sites $^{25}$. Equal amounts of DpnI-digested DNA and undigested control DNA were subjected to quantitative polymerase chain reaction (qPCR) analyses with primers targeting $6 \mathrm{~mA}$ dynamic regions identified by $6 \mathrm{~mA}-\mathrm{IP}$. The percentage of $6 \mathrm{~mA}$ in either control or stressed PFC can be assessed by qPCR amplification and normalized to undigested DNA control (digested/undigested) ${ }^{4}, 25$. Loci with lower $6 \mathrm{~mA}$ modification upon stress would hinder $D p n I$ digestion, resulting in higher PCR fold changes than control. We validated 16 random gain- or loss-of- $6 \mathrm{~mA}$ loci annotated to intragenic or intergenic regions identified by $6 \mathrm{~mA}-\mathrm{IP}$-seq and found consistent $6 \mathrm{~mA}$ dynamic changes upon stress (Supplementary Figs. 2, 3a, b). Furthermore, we confirmed four additional loss-of- $6 \mathrm{~mA}$ regions annotated in genes involved in neurodevelopment and behavior (Supplementary Fig. 3c). Importantly, no significant difference was found in random loci without $6 \mathrm{~mA}$ changes, indicating the reproducibility and reliability of $6 \mathrm{~mA}$ differential region identification (Supplementary Fig. 3d). However, although DpnI can cleave fully methylated adenine at GATC/CATC/CATG to indicate specific methylated adenine in these sites, methylated adenines outside these cutting sites are beyond the scope of DpnIbased $6 \mathrm{~mA}$ detection, which we could not validate here. Finally, we confirmed differential $6 \mathrm{~mA}$ regions by an additional computational package (DESeq 2$)^{26}$, and found substantial and significant overlap between $6 \mathrm{~mA}$ differential regions identified by DESeq 2 and edgeR that was initially used to identify differential $6 \mathrm{~mA}$ regions. Taken together, these analyses confirm dynamic $6 \mathrm{~mA}$ regions upon stress identified by $6 \mathrm{~mA}-\mathrm{IP}$-seq.

$N(6)$-methyladenine negatively correlates with a group of neuronal gene expression. To further link $6 \mathrm{~mA}$ dynamics and gene expression, global transcriptome changes in PFC upon stress were assessed by RNA-seq (Supplementary Data 4). Gene 
a

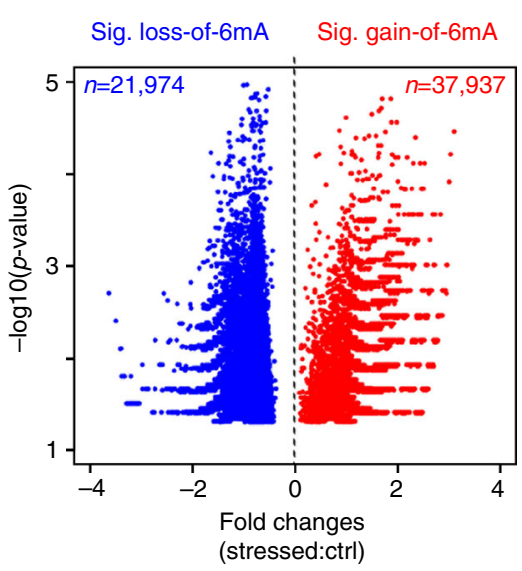

C

Sig.loss-of-6mA regions annotation

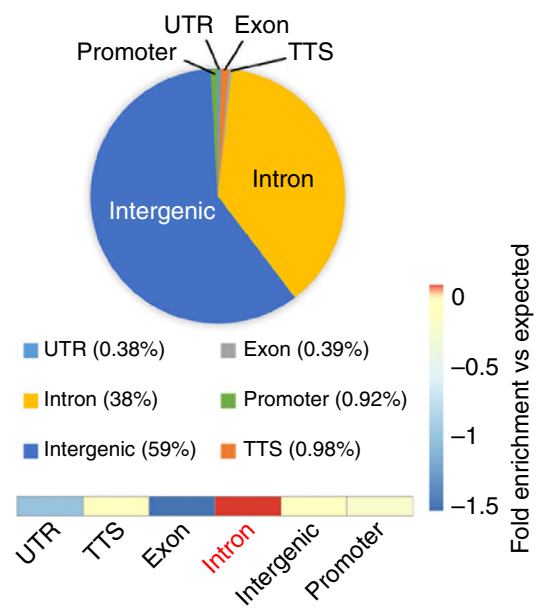

b

Sig. gain-of-6mA regions annotation

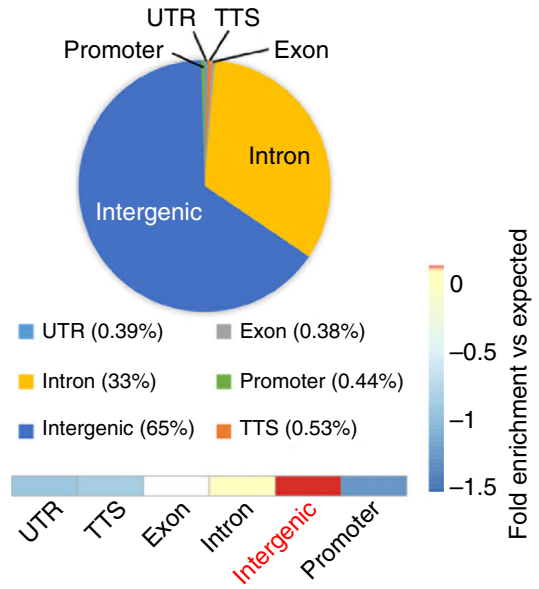

d

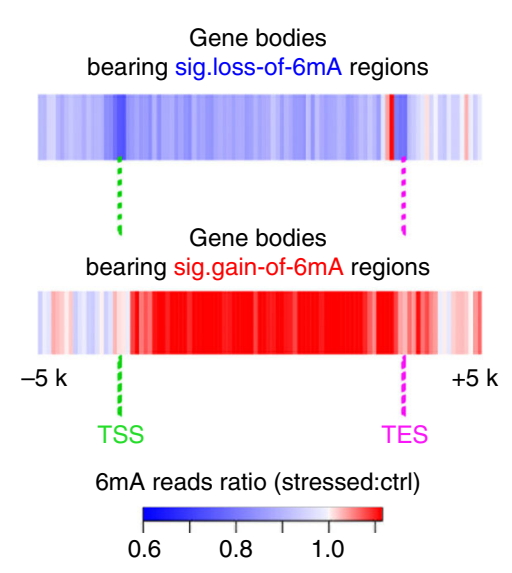

Fig. 2 Genome-wide $6 \mathrm{~mA}$ profiling in PFC reveals dynamic changes in $6 \mathrm{~mA}$ on intragenic regions in response to stress. a Volcano plot illustrated the significant gain- and loss-of- $6 \mathrm{~mA}$ regions upon stress. Each dot represents 500-bp binned mouse genome containing significant $6 \mathrm{~mA}$ reads differences by pair-wise replicate comparison. Fold changes in $6 \mathrm{~mA}$ reads (Stressed:Control) are indicated on the $x$-axis, and - $\log 10(p$-value) of each bin are indicated on the $y$-axis. 37,937 significant gain-of- $6 \mathrm{~mA}$ regions and 21,974 loss-of- $6 \mathrm{~mA}$ regions are highlighted in red and blue, respectively. b, c Genomic annotation of significant gain- or loss-of- $6 \mathrm{~mA}$ demonstrate their percentage of genomic feature association and enrichment vs. expected values. Dynamic $6 \mathrm{~mA}$ changes upon stress predominantly occurred on introns and intergenic regions. Gain-of- $6 \mathrm{~mA}$ regions were mostly enriched in intergenic regions over expected value whereas loss-of-6mA enriched in introns over expected values (Heatmap view, highlighted in red). d Average fold change between $6 \mathrm{~mA}$ normalized reads (stressed vs. control) were calculated in gene bodies bearing loss- or gain-of $6 \mathrm{~mA}$ regions upon stress, respectively, plus $5 \mathrm{~kb}$ upstream and downstream flanking regions. Average fold change was plotted in Heatmap view. Red plots (fold change $>1$ ) indicate overall gain of $6 \mathrm{~mA}$ upon stress whereas blue plots (fold change $<1$ ) indicate loss of $6 \mathrm{~mA}$

Ontology (GO) analyses revealed enrichment for numerous biological pathways involved in neuronal functions in the cohort of upregulated genes (Supplementary Fig. 4a). In contrast, these terms were not found in the GO analysis of downregulated genes (Supplementary Fig. 4b), indicating that many critical neuronal genes were upregulated in response to stress. To investigate the potential roles of $6 \mathrm{~mA}$ in the stress response, we computed the overall $6 \mathrm{~mA}$ reads ratio at significant intragenic gain- or loss-of$6 \mathrm{~mA}$ regions per gene between stressed and control PFC, and further subcategorized these genes into four groups: gain-of$6 \mathrm{~mA} /$ upregulated, gain-of- $6 \mathrm{~mA} /$ downregulated, loss-of- $6 \mathrm{~mA} /$ upregulated, and loss-of-6mA/downregulated genes. Importantly, GO analyses revealed that only upregulated genes bearing significant loss-of-6mA were strongly associated with biological pathways (top 15 terms based on fold enrichment) related to neuronal development and neuronal functions, such as learning, behavior, neurogenesis, and axon development, which have been shown to play important roles in the brain stress response $\mathrm{e}^{27,28}$ (Fig. 4a, b and Supplementary Data 5). Cross-examination of the relationship between genes and GO terms suggested that a substantial number of upregulated genes related to behavior were also enriched for other biological functions such as learning and neuron projection development, suggesting that these genes could be involved in multiple biological pathways orchestrating stress response (Fig. 4c). On the other hand, no biological terms related to neuronal functions were found in the other groups of genes (Supplementary Fig. 5a-c). Thus, our findings suggest a specific inverse association between intragenic loss-of- $6 \mathrm{~mA}$ and a set of critical upregulated neuronal genes in response to stress. This data echoes the observation that loss-of- $6 \mathrm{~mA}$ regions were 
a

Intergenic sig. gain-of-6mA annotation

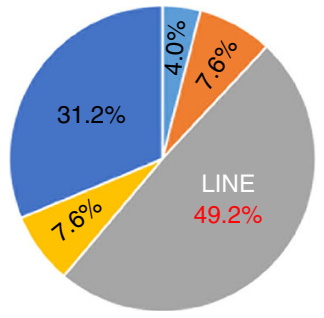

C

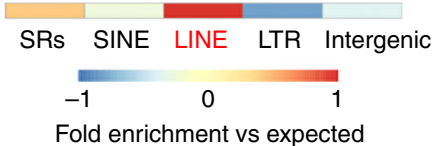

b

Intergenic sig. loss-of-6mA annotation

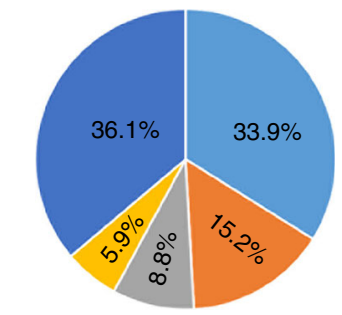

d

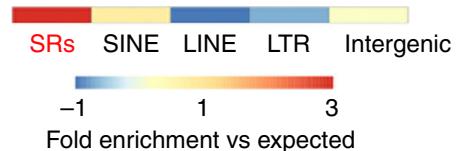

e

LINE transposon (103/120 total expressed) with sig. $6 \mathrm{~mA}$ alteration upon stress

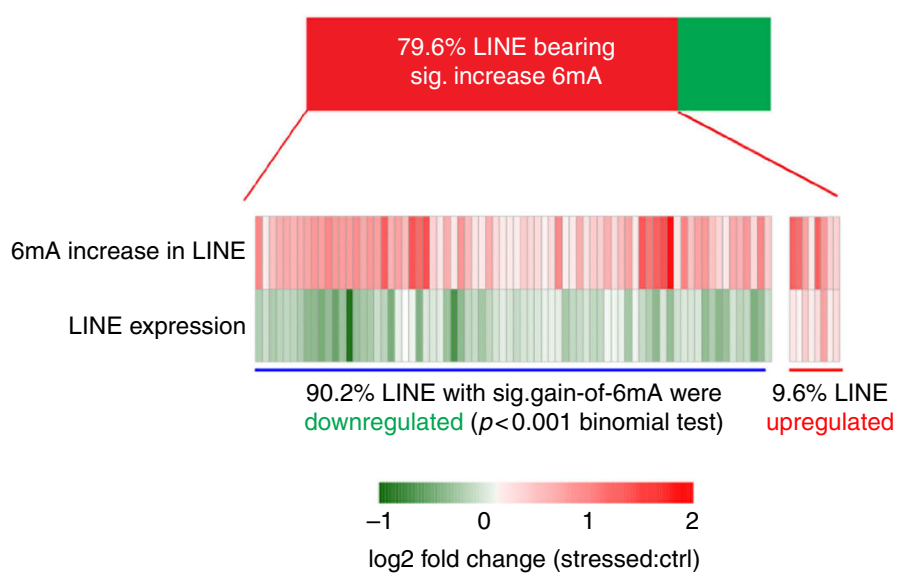

Fig. 3 Genome-wide $6 \mathrm{~mA}$ profiling in PFC reveals dynamic changes in $6 \mathrm{~mA}$ on intergenic repetitive elements in response to stress. $\mathbf{a}$, $\mathbf{b}$ Genomic annotation of significant gain- or loss-of- $6 \mathrm{~mA}$ demonstrate their percentage of repetitive elements association and enrichment vs. expected values. Stressinduced intergenic $6 \mathrm{~mA}$ alterations occurred on distinct classes of repetitive elements. $\mathbf{c}$, $\mathbf{d}$ The Log2 enrichment of annotated gain- or loss-of- $6 \mathrm{~mA}$ vs. expected values were calculated by HOMER annotation algorithm and demonstrated by heatmap. In total, $49.2 \%$ of intergenic gain-of- $6 \mathrm{~mA}$ occurred in the long interspersed nuclear element (LINE), which was highly enriched over expected. Loss-of- $6 \mathrm{~mA}$ was predominantly enriched in simple repeats over expected values. e Gain-of-6mA on intergenic regions correlated with downregulation of LINE transposon expression. In total, $79.6 \%$ of dynamic $6 \mathrm{~mA}$ marked LINE transposons possessed gain-of-6mA, with $90.4 \%$ of these LINE expression being downregulated upon stress. $P<0.001$, Chi-squared test comparing to short interspersed nuclear element (SINE) transposon. The fold changes (Stressed:Control) of both $6 \mathrm{~mA}$ and LINE expression are indicated by Heatmap. Red plots (Log2 fold change $>0$ ) indicate increased expression upon stress (stressed $>$ control), whereas green plots (fold change $<0$ ) indicate decreased expression upon stress (stressed $<$ control)

strongly and uniquely enriched at introns relative to expected values (Fig. 2c).

Crosstalk between $6 \mathrm{~mA}$ and other epigenetic mechanisms. In order to explore the mechanistic roles of $6 \mathrm{~mA}$ in gene regulation, we investigated the potential correlation between $6 \mathrm{~mA}$ distribution and enhancer regions and their signature histone modifications ${ }^{29}$. Overall, $6 \mathrm{~mA}$ levels in both control and stressed PFC samples appeared to be significantly depleted in both general enhancers and cortex-specific enhancers compared to nonenriched input (Supplementary Fig. 6a). Stress further significantly decreased $6 \mathrm{~mA}$ levels in these regions, implying that altered $6 \mathrm{~mA}$ upon stress could impact enhancer activity in mouse brains (Supplementary Fig. 6b). H3K27ac and H3K4me1 are associated with enhancers and active promoters ${ }^{29}$. Consistent with enhancer regions, $6 \mathrm{~mA}$ was also depleted at $\mathrm{H} 3 \mathrm{~K} 27 \mathrm{ac}$ and $\mathrm{H} 3 \mathrm{~K} 4 \mathrm{mel}^{30}$ regions (Supplementary Fig. $6 \mathrm{c}$ ). In addition, $6 \mathrm{~mA}$ was also depleted at Pol II binding sites, consistent with its negative correlation with transcription. Interestingly, although the same trend (further $6 \mathrm{~mA}$ depletion upon stress) was observed in $\mathrm{H} 3 \mathrm{~K} 27 \mathrm{Ac}$ and H3K4me1, stress-induced $6 \mathrm{~mA}$ depletion was not significant (Supplementary Fig. 6d). This observation suggested that $6 \mathrm{~mA}$ dynamic changes in response to stress were more specific at cis-regulatory regions.

Recent studies suggest that DNA methylation, especially noncanonical $\mathrm{CpH}$ methylation $(\mathrm{H}=\mathrm{A}, \mathrm{C}$ or $\mathrm{T})$, plays unique roles in neuronal functions ${ }^{31}, 32$. We explored the correlation between cytosine methylation and adenine methylation in the context of neurons and non-neurons using a genome-wide methylated DNA immunoprecipitation (MeDIP) data set in mouse cortex ${ }^{33}$. Interestingly, methylated cytosine $(\mathrm{mC})$ was strongly enriched at loss-of-6mA regions but only showed modest distributions at gain-of-6mA regions (Supplementary Fig. 7a). The specific enrichment of $\mathrm{mC}$ at regions with high $6 \mathrm{~mA}$ levels in control, 
which lose $6 \mathrm{~mA}$ under stress, could indicate co-existence of these modifications and coordination of repressive roles. Intriguingly, the correlation between $\mathrm{mC}$ and $6 \mathrm{~mA}$ appears to be preferential in neurons, as loss-of-6mA regions have almost two-fold higher $\mathrm{mC}$ levels in neurons than non-neuronal cells (Supplementary Fig. 7b). Consistently, global $6 \mathrm{~mA}$ profiles in control PFC demonstrated a stronger correlation with $5 \mathrm{mC}$ than stressed PFC, and the $5 \mathrm{mC}$ profile was closer to $6 \mathrm{~mA}$ profiles in neuronal cells than non-neuronal cells (Supplementary Fig. 8a). Given that a substantial number of gain-of-6mA regions upon stress were annotated in LINE transposons, we tested whether cytosine methylation was also altered in LINE transposons. Interestingly, MeDIP coupled with $\mathrm{qPCR}$ detected a significant increase of methylation in $\mathrm{L}_{1 \mathrm{td}} 1^{34}$ upon stress (Supplementary Fig. 8b), suggesting potential crosstalk between $6 \mathrm{~mA}$ and $5 \mathrm{mC}$ in LINE transposons. Furthermore, the correlation between cytosine and adenine methylation was supported by the top motifs found in intragenic regions upregulated with loss-of-6mA (Supplementary Fig. 9). "AC" and "CA" are major motifs within these regions in PFC. Moreover, these motifs were predicted to be recognized by various transcription factors, such as Egr2, Foxh1 and Hif2a (Supplementary Fig. 9), suggesting that $6 \mathrm{~mA}$ dynamics may serve a

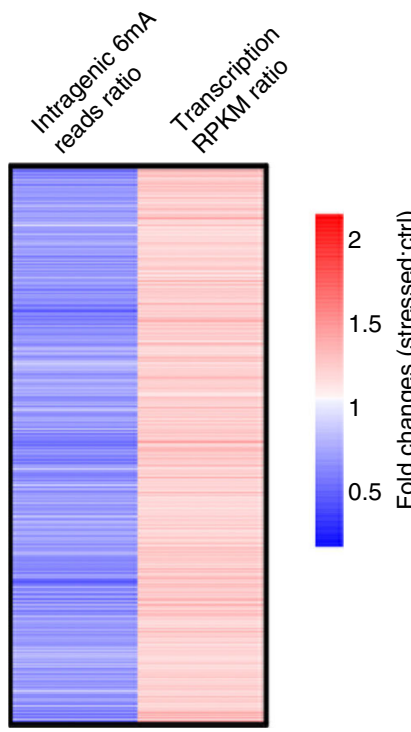

b
C

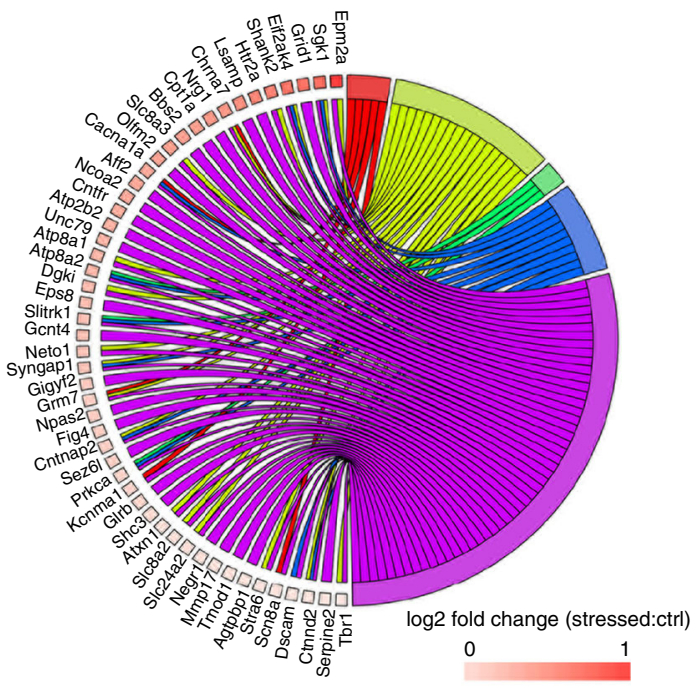

GO: Behavior

GO: Neuron projection development

GO: Learning

GO: Transmission of nerve pulse

GO: Axongenesis

Loss-of-6mA/upregulated genes top $10 \mathrm{GO}$ terms

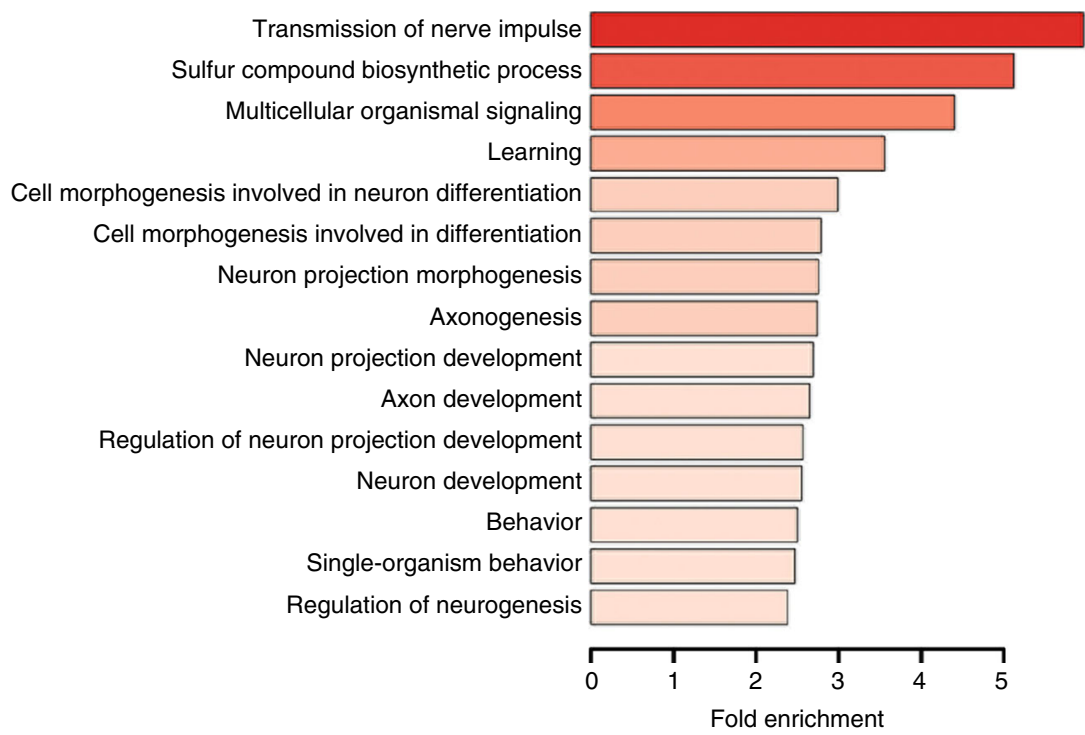

Fig. 4 N(6)-methyladenine negatively correlates with neuronal gene expression in response to stress. a Genes with significant loss-of-6mA and increased expression upon stress are indicated. The log2 fold changes (Stressed:Control) of both $6 \mathrm{~mA}$ and transcription are illustrated by Heatmap. Red plots (fold change $>1$ ) indicate transcription upregulation upon stress, whereas blue plots (fold change $<1$ ) indicate loss-of- $6 \mathrm{~mA}$ on the same genes upon stress. b Genes in a were enriched in pathways related to neuronal functions, neurogenesis and behavior control. Fold enrichment of each $\mathrm{GO}$ term are indicated by the $x$-axis and bar color. c Circos plot to indicate the relationship between genes and GO terms. Cross-examination of GO analyses suggested that a substantial number of upregulated genes with loss-of- $6 \mathrm{~mA}$ related to behavior were also enriched in other biological functions such as learning and neuron projection development. Log2 fold changes of gene expression were indicated as colored squares 


\section{a}
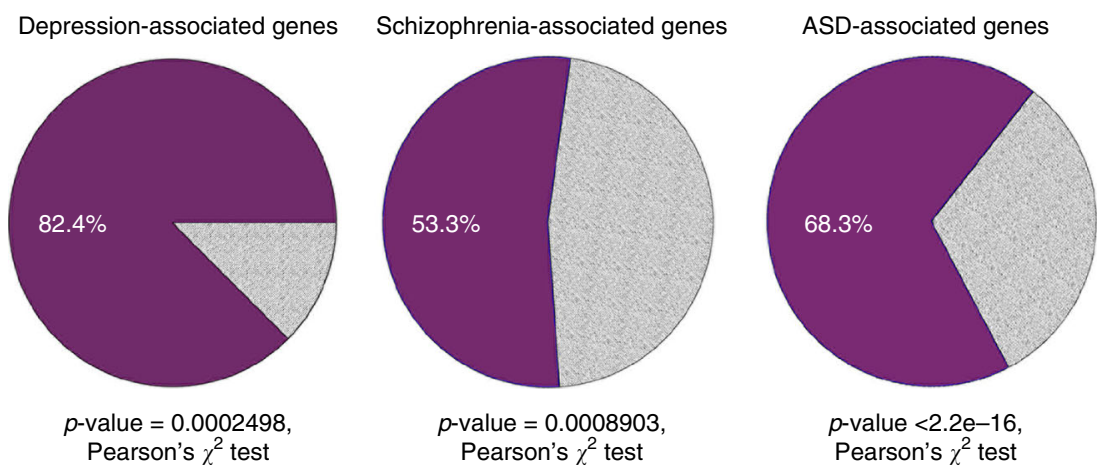

\section{b}
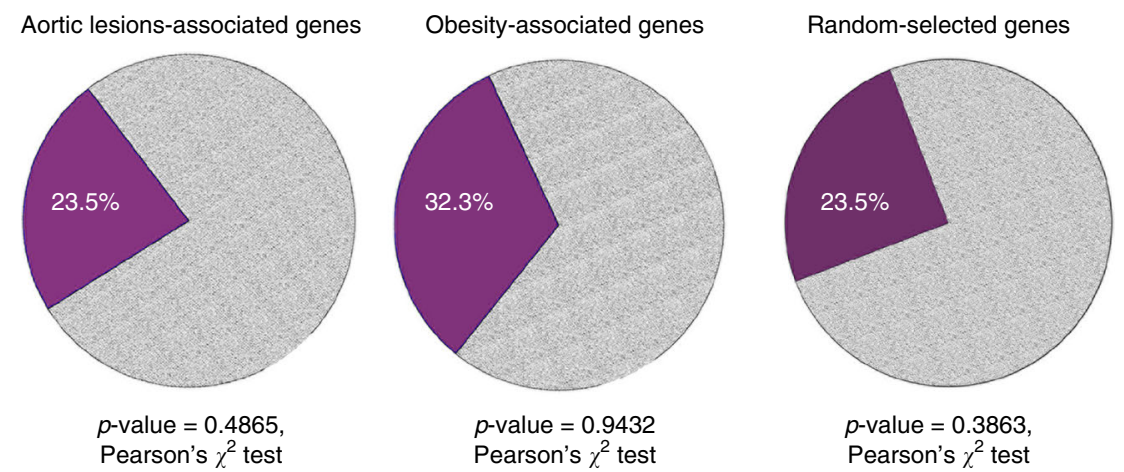

Genes with sig.

$6 \mathrm{~mA}$ changes

Genes without sig.

$6 \mathrm{~mA}$ changes

Fig. 5 Dynamic 6mA is associated with depression-linked genetic loci. a Significant overlap between depression-associated genes containing the top 17 most significant SNPs, schizophrenia (SCZ)-related risk genes and autism spectrum disorders (ASD)-related risk genes with dynamic $6 \mathrm{~mA}$ marked genes upon stress are indicated. P-value was calculated by Pearson's $\chi^{2}$ test. $\mathbf{b}$ The genes related to aortic lesions, obesity or randomly selected genes did not show significant enrichment. Statistical significance was calculated by Pearson's $\chi^{2}$ tests, and $p$-values are indicated

as docking sites to modulate transcription factor binding in response to stress. In fact, $6 \mathrm{~mA}$ is enriched in histone modifier binding sites, such as Polycomb PRC1 component BMI1 ProtoOncogene, Polycomb Ring Finger (Bmi1), but depleted in another Polycomb protein RING1B (Supplementary Fig. 10a), implying potential and specific crosstalk between $6 \mathrm{~mA}$ and other epigenetic mechanisms. Significant loss-of- $6 \mathrm{~mA}$ in stress was found at Bmil-binding sites (Supplementary Fig. 10b), and Bmil has been shown to mark certain enhancer regions ${ }^{35}$. This observation was consistent with the depletion of $6 \mathrm{~mA}$ from enhancers in normal mouse PFC (Supplementary Fig. 6).

Dynamic $6 \mathrm{~mA}$ is associated with depression-linked loci. To shed light on the functional relevance of $6 \mathrm{~mA}$ dynamics in the stress response and human neuropsychiatric disorders, we obtained human depression-associated genes related to the 17 most significant $\mathrm{SNPs}^{36}$, and correlated their mouse orthologs with $6 \mathrm{~mA}$ dynamic genes upon stress in our study. Interestingly, 14 out of $17(82.4 \%)$ depression-associated genes significantly overlapped with $6 \mathrm{~mA}$ dynamic genes upon stress (Fig. 5a, Pearson's $\chi^{2}$ tests). Importantly, the same number of random genes selected to match the GC content, gene size, and neuronal expression of these depression-associated genes did not significantly overlap with $6 \mathrm{~mA}$ dynamic genes upon stress (Supplementary Fig. 11). To further investigate whether $6 \mathrm{~mA}$ is associated with other neuropsychiatric disorders, we obtained genes associated with autism spectrum disorder $(\mathrm{ASD})^{37}$, schizophrenia
$(\mathrm{SCZ})^{38}$, aortic lesion, and obesity ${ }^{39} . N(6)$-methyladenine dynamic genes showed significant and specific overlap with ASD and SCZ genes (Fig. 5a, Pearson's $\chi^{2}$ test), but not with genes involved in aortic lesion, obesity ${ }^{39}$, or an equal number of randomly selected refseq genes (Fig. 5b, Pearson's $\chi^{2}$ test). Together, these data suggest a potential link between this novel DNA modification and mental illness. Furthermore, genes with the top 10,000 depressionassociated SNPs also displayed significant overlap with $6 \mathrm{~mA}$ dynamic genes but not with an equal number of random genes (Supplementary Fig. 12a, Pearson's $\chi^{2}$ tests). We generated three random gene lists to match the GC content, gene size, and neuronal expression to the full list of depression-associated genes, and calculated their overlap with $6 \mathrm{~mA}$ dynamic genes in stress (Supplementary Fig. 12b-d). Similar to the top 17 most significant SNPs, the overlap between $6 \mathrm{~mA}$ dynamic genes in stress and the full list of depression-associated genes is also specific and significant, as all three random gene lists did not show significant overlap with $6 \mathrm{~mA}$ dynamic genes (Supplementary Fig. 12b-d, Pearson's $\chi^{2}$ tests). These data indicate a strong association between $6 \mathrm{~mA}$ and loci linked to neuropsychiatric disorders.

\section{Discussion}

$N(6)$-methyladenine is relatively well characterized in bacteria and is involved in host defense against bacteriophages and transposons, modulation of DNA and chromosome replication, DNA mismatch repair and expression of certain operons ${ }^{3}$. The presence of $6 \mathrm{~mA}$ in the genome of eukaryotic cells, including 
green algae, worms, and fruit flies, was recently documented ${ }^{4-6}$. Our data presented here for the first time demonstrate the presence and dynamics of $6 \mathrm{~mA}$ in the mouse brain in response to stress. Genome-wide $6 \mathrm{~mA}$ profiling indicates an inverse association of $6 \mathrm{~mA}$ dynamics with a group of neuronal genes involved in the stress response as well as transposon LINE expression. $N(6)$ methyladenine appears to be depleted in cis-regulatory elements and correlates with cytosine methylation and other epigenetic regulation in the mammalian CNS. Interestingly, the group of genes in mouse PFC bearing dynamic changes in $6 \mathrm{~mA}$ in response to stress significantly and specifically associate with genes involved in neuropsychiatric disorders.

Increasing evidence indicate that physiological and psychological stress could change DNA methylation at critical genes related to stress in mouse brains ${ }^{18,40,41}$. Furthermore, DNA methylation alterations are further supported by similar observations in postmortem human brains of patients diagnosed with major depressive disorder who committed suicide ${ }^{42}$. PFC is responsible for highest-order cognitive abilities and is particularly susceptible to chronic stress and plays significant roles in depression $^{22}$. Our results demonstrate specific accumulation of $6 \mathrm{~mA}$ in PFC under chronic stress, which further supports the critical roles of PFC in the stress response and suggest the potential importance of $6 \mathrm{~mA}$ dynamics during this process. Interestingly, it has been suggested that chronic stress could induce morphological and molecular changes within PFC to render cognitive rigidity and increased vigilance ${ }^{28}$. These changes are PFC specific, and could be conveyed by the rapid alteration of $6 \mathrm{~mA}$ upon stress. It is important to note that the stress condition applied in our current study only represents one of many unknown environmental paradigms which could spatially and temporally trigger $6 \mathrm{~mA}$ dynamics to deal with environmental changes at the epigenetic level. Thus, further studies will be important to precisely elucidate the roles of $6 \mathrm{~mA}$ in specific brain regions in response to various stimuli.

Earlier experiments have found that transfecting a gene with $6 \mathrm{~mA}$ modifications could result in an almost 30 -fold decrease in transcription factor binding in mammalian cells, implying potential roles for $6 \mathrm{~mA}$ in transcription ${ }^{43}$. In the current study, we show that significant loss of intragenic $6 \mathrm{~mA}$ in mouse PFC upon stress inversely correlates with a group of upregulated genes enriched in neuronal functions, neurodevelopment and behavior. These data indicate that the presence of $6 \mathrm{~mA}$ on these genes could play a negative transcription role under normal conditions in mouse PFC, consistent with a recent study in mESCs ${ }^{9}$. Interestingly our data suggest a general depletion of $6 \mathrm{~mA}$ in enhancer regions and Pol II binding sites (Supplementary Fig. 5c), indicating that $6 \mathrm{~mA}$ repression could be in part due to its modulation of intronic cis-regulatory regions. Genome-wide $6 \mathrm{~mA}$ distribution appears to be tissue-specific, as dynamic $6 \mathrm{~mA}$ changes are highly enriched in "CA" motifs in mouse PFC compared to "GA" motifs in static mouse kidney ${ }^{7}$. Increasing evidence supports that non$\mathrm{CpG}$ methylation ( $\mathrm{mCH}, \mathrm{H}=\mathrm{A} / \mathrm{C} / \mathrm{T}$ ) more directly regulates genes in neurons, with $\mathrm{mC}$ in the "CA" context as a major motif $^{32}$. It is important to further understand the precise and tissue-specific roles of $6 \mathrm{~mA}$ and investigate the potential link between cytosine and adenine methylation.

Our finding that the group of genes bearing dynamic $6 \mathrm{~mA}$ changes in response to stress significantly and specifically overlaps with genes involved in depression, SCZ, and ASD is particularly intriguing. Potential 6mA "readers", "writers", and "erasers" that maintain $6 \mathrm{~mA}$ homeostasis in the CNS could play key roles in maintaining normal brain function. It will be important to identify the $6 \mathrm{~mA}$ functional players in future studies. Thus, aberrant $6 \mathrm{~mA}$ in response to stress could contribute to neuropsychiatric diseases by ectopically recruiting transcription factors and altering target expression. Therefore, our finding provides the first link between this novel DNA adenine methylation and neuropsychiatric disorders.

\section{Methods}

Animals. All wild-type mice had the C57BL/6 genetic background. All mice used in this study were male and 7-8 weeks old. All animal procedures were performed according to protocols approved by Institutional Animal Care and Use Committee at Emory University.

Chronic restraint stress. Mice were individually placed into a customized, wellventilated 50-ml conical tube (Corning Inc., Corning, NY) daily from 10 a.m. to $12 \mathrm{p} . \mathrm{m}$. for $2 \mathrm{~h}$ in their home cages for 2 weeks. The restrained mice could move from the supine to prone position, but were not able to move forward or backward The non-restrained control mice remained undisturbed in their home cages. After restraint stress, mice were released from the tube and returned to their home cages. Three independent stress experiments were performed with nine animals each in control and stressed groups per experiment.

Forced swim test. The FST was performed as described previously ${ }^{44}$. In brief, each mouse was placed in a glass cylinder $(20 \mathrm{~cm}$ high, $15 \mathrm{~cm}$ in diameter) with warm water $\left(23-25^{\circ} \mathrm{C}, 14 \mathrm{~cm}\right.$ in depth) for $6 \mathrm{~min}$. The water in the glass cylinder was replaced with fresh water of the same temperature for each individual test. Mice were gently dried after removal from the bath and returned to their home cages. Immobility time was recorded when the animal was floating in the water or making the minimum movement necessary to float in the water. An unpaired $t$-test was used and error bars represente mean \pm SEM. Investigators were blinded to groups and genotypes.

Tail suspension test. The TST was performed according to the method outlined in previous reports with a minor modification ${ }^{45}$. Each mouse was suspended by adhesive tape placed $\sim 1 \mathrm{~cm}$ from the tip of the tail. The distance between the tip of the tail of each mouse and the desktop was about $45 \mathrm{~cm}$. Each mouse was suspended for $6 \mathrm{~min}$, and the immobility time was recorded. Unpaired $t$-test was used and error bars represent mean \pm SEM. Investigators were blinded to groups and genotypes.

Genomic DNA and RNA isolation. Mouse prefrontal cortexes were dissected from the brain immediately after the behavior tests. Genomic DNA was isolated from different brain tissues in $600 \mu \mathrm{l}$ of digestion buffer $(100 \mathrm{mM}$ Tris- $\mathrm{HCl}, \mathrm{pH} 8.5$, $5 \mathrm{mM}$ EDTA, $0.2 \%$ SDS, $200 \mathrm{mM} \mathrm{NaCl}$ ), then treated with Proteinase $\mathrm{K}$ (Thermo) at $55^{\circ} \mathrm{C}$ overnight. The second day, $600 \mu \mathrm{l}$ of phenol:chloroform:isoamyl alcohol (25:24:1 saturated with $10 \mathrm{mM}$ Tris, $\mathrm{pH}$ 8.0, 1 mM EDTA) (P-3803, Sigma) was added to samples, mixed completely, and centrifuged for $10 \mathrm{~min}$ at $12,000 \mathrm{rpm}$. The aqueous layer was transferred into a new Eppendorf tube and precipitated with $600 \mu \mathrm{l}$ of isopropanol. The pellet was washed with $75 \%$ ethanol, air-dried, and resuspended with Nuclease-Free Water (Ambion). DNA samples were treated extensively with an RNase cocktail (Ambion) to remove contaminating RNA. PFC samples were homogenized in Trizol (Invitrogen) and processed according to the manufacturer's instructions for total RNA isolation.

N(6)-methyladenine antibody specificity. The specificity of $6 \mathrm{~mA}$ antibody (Synaptic systems) was extensively validated to ensure that it did not cross-react with $5 \mathrm{mC}, 3 \mathrm{~mA}$, or denatured $1 \mathrm{~mA}^{5}$. It displayed poor reactivity with RNA molecules ${ }^{5}$. In addition, consistent with our data, a recent publication ${ }^{9}$ used this LC-MS approach to analyze DNA modifications in mouse embryonic stem cells. No 1-methyladenine $(1 \mathrm{~mA})$, 3-methyladenine $(3 \mathrm{~mA})$, or 3-methyl-cytosine $(3 \mathrm{mC})$ was detected in the mouse genome. The specificity of this antibody was validated by PCR using normal dATP or 6mA-modified dATP (Fig. 1d).

N(6)-methyladenine dot blot. Dot blot was performed on a Bio-Dot Apparatus (\#170-6545, BIO-RAD) as described previously ${ }^{46}$ using a specific $6 \mathrm{~mA}$ antibody (Synaptic Systems, cat. no. 202003 ) incubating overnight at $4{ }^{\circ} \mathrm{C}$. Horseradish peroxidase-conjugated antibody to rabbit (1:5000, \#A-0545, Sigma) was used as a secondary antibody, and incubated for $45 \mathrm{~min}$ at $20-25^{\circ} \mathrm{C}$. Standard DNA templates were loaded (D5405, ZYMO) for quantification and to verify antibody specificity. The density of each dot signal was quantified by ImageJ software ${ }^{47}$. An unpaired $t$-test was used and error bars represent mean \pm SEM. Extensive RNase treatments were performed to ensure complete removal of RNA contamination.

UHPLC-MS/MS analysis. Genomic DNA of PFC, HIP, AMY, and HYP were enzymatically digested into single nucleosides with a mixture of DNaseI, calf intestinal phosphatase, and snake venom phosphodiesterase I at $37^{\circ} \mathrm{C}$ for $12 \mathrm{~h}$. After enzymes were removed by ultrafiltration, the digested DNA was subjected to UHPLC-MS/MS analysis. HPLC fractionation of mouse m6dA and UHPLC-

QTOF-MS/MS analysis were performed as described previously ${ }^{6}$. The $6 \mathrm{~mA}$ levels 
in control PFC (6.6 p.p.m.) and stressed PFC (25.5 p.p.m.) had similar ranges as observed in mouse embryonic stem cells ${ }^{9}$.

N(6)-methyladenine immunoprecipitation. Genomic DNA was sonicated to 200-300-bp fragments for IP-based $6 \mathrm{~mA}$ enrichment using rabbit polyclonal antibody raised against $6 \mathrm{~mA}$ (Synaptic Systems) at 1:100 in 1× IP buffer containing $100 \mathrm{Mm}$ Tris- $\mathrm{HCl} \mathrm{pH}$ 7.4, $150 \mathrm{Mm} \mathrm{NaCl}, 0.05 \%$ Triton X-100. The IP was conducted with overnight platform rotation at $4{ }^{\circ} \mathrm{C}$ without beads. Dynabeads Protein G (Novex by Life Technologies, REF 10009D $30 \mathrm{mg} / \mathrm{ml}$ ) were added, and the mixture was rotated at $4{ }^{\circ} \mathrm{C}$ for $2 \mathrm{~h}$. Beads were washed six times at room temperature with $1 \times$ ice-cold IP buffer, 5 min each with rotation. After the wash, the DNA fragments were eluted by either proteinase $\mathrm{K}$ digestion or $6 \mathrm{~mA}$ competition. For proteinase $\mathrm{K}$ digestion-based elution, the beads were treated with digestion buffer containing $1 \times \mathrm{TE}, 0.25 \% \mathrm{SDS}$, and $0.25 \%$ Proteinase $\mathrm{K}(2.5 \mathrm{mg} / \mathrm{ml})$ at $55^{\circ} \mathrm{C}$ and centrifuged at $1400 \mathrm{rpm}$ for $2 \mathrm{~h}$. The eluted DNA fragments were extracted once with phenol:chloroform:isoamyl alcohol=25:24:1, followed by extraction with chloroform alone, and then precipitated with glycogen, $\mathrm{NaOAc} \mathrm{pH}$ 5.2, and $100 \%$ ethanol at $-20^{\circ} \mathrm{C}$ for at least $2 \mathrm{~h}$ or overnight. For $6 \mathrm{~mA}$ base competition-based elution, the beads were treated with $1 \times$ IP buffer with $2.6 \mathrm{mM} 6 \mathrm{~mA}$ three times for $30 \mathrm{~min}$ each at room temperature. The eluted DNA fragments were precipitated without extraction with phenol and chloroform.

N(6)-methyladenine Dpnl digestion and qPCR. DpnI digestion and qPCR were conducted as previously described ${ }^{25}$. Briefly, restriction enzyme digestion was performed by treating $1 \mu \mathrm{g}$ of genomic DNA with $5 \mu \mathrm{l}$ of $5 \mathrm{U} / \mu \mathrm{l} D p n \mathrm{I}$ restriction enzyme (NEB) at $37^{\circ} \mathrm{C}$ for $1 \mathrm{~h}$. The digested DNA and non-digested DNA (5 ng) were subjected to qPCR using FastStart SYBR Green Master kit.

\section{Library preparation and high-throughput sequencing. Enriched DNA from} triplicate 6mA-IP were subjected to library construction using the NEBNext ChIPSeq Library Prep Reagent Set from Illumina according to the manufacturer's protocol. Briefly, $25 \mathrm{ng}$ of input genomic DNA or experimental enriched DNA were used for each library construction. DNA fragments (150-300 bp) were selected by AMPure XP Beads (Beckman Coulter) after adapter ligation. An Agilent 2100 BioAnalyzer was used to quantify amplified DNA, and qPCR was applied to accurately quantify library concentration. In total, $20 \mathrm{pM}$ diluted libraries were used for sequencing. Fifty cycle single-end sequencing reactions were performed using Illumina HiSeq 2000. Image processing and sequence extraction were done using the standard Illumina Pipeline.

RNA-seq libraries were generated from triplicate samples per condition using the Illumina TruSeq RNA Sample Preparation Kit v2 following the manufacturer's protocol. RNA-seq libraries were sequenced as 50 -cycle paired-end runs using Illumina HiSeq 2000.

Sample size and statistical analyses. Eighteen wild-type mice were randomly divided into control and stressed groups. No particular animals were included or excluded from the study. For 6mA profiling, three biological replicates (both control and stressed mouse PFC DNA) from different batches of stress experiments with consistent PFC 6mA increases were subjected to 6mA-IP-seq. RNA-seq was also performed on three biological replicates. $P$ values from Pearson's $\chi^{2}$ tests and $t$ tests are indicated in Figures and Figure legends. All statistical analyses were performed in the $\mathrm{R}$ computational environment.

Bioinformatics analyses. Triplicated FASTQ sequence files from control and stressed $6 \mathrm{~mA}$-IP in PFC were aligned to the $\mathrm{mm} 9$ reference genome using Bowtie v1.1.2 ${ }^{48}$. Established computational algorithms (edgeR package in R/Bioconductor environment) using quantile-adjusted conditional maximum likelihood methods were used to compare three control and three stressed normalized $6 \mathrm{~mA}$ read density in $500 \mathrm{bp}$ binned mouse genome (6mA-seq resolution) with the consideration of sample variation. Differential regions with a $p$ value $<0.05$ (false discovery rate $<0.077$ ) were considered significant. The differential $6 \mathrm{~mA}$ regions were further validated by DESeq2, another widely utilized computational algorithm ${ }^{26}$. Ngs.plot software was used to calculate $6 \mathrm{~mA}$ and $5 \mathrm{mC}$ concatenated reads on various genomic regions to plot average $6 \mathrm{~mA}$ reads on gene bodies ${ }^{49}$. Annotation analyses and expected values of genomic features were performed using the HOMER suite ${ }^{50}$. Motif search and transcription factor prediction were performed by regulatory sequence analysis tools ${ }^{51}$ or HOMER suite. RNA-seq reads were aligned using TopHat v2.0.13 million mapped reads (RPKM) expression values were extracted using Cuffdiff v2.2.1 ${ }^{52}$. Genes with RPKM values more than 0.5 and $\log 2$ fold changes more than 0.1 were kept for transcriptome analyses. All differentially expressed genes were tested for correlation to $6 \mathrm{~mA}$ dynamic changes since stress could result in subtle changes in expression. Gene ontology analyses were performed by Gene Ontology Consortium ${ }^{53}$. Transposon expression was analyzed by TEtranscripts ${ }^{54}$. Circos plot was generated by R-package GOplot ${ }^{55}$. The global Spearman correlations between $6 \mathrm{~mA}$ and $5 \mathrm{mC}$ were performed by deepTools ${ }^{56}$.
Published datasets. Cortex-specific enhancer and cortex general enhancers were obtained from the ENCODE project ${ }^{29}$ (http://chromosome.sdsc.edu/mouse/ download.html). Enhancer regions were determined as $250 \mathrm{bp}$ flanking the summit. Eight-week-old mouse cortex H3K27ac and h3K4mel ChIP-seq data were obtained from GSE $49847^{29}$. Mouse anterior cingulate cortex (ACC) DNA methylation (MeDIP-seq), Histone H3K27ac, and H3K4mel ChIP-seq data were obtained from GSE74965 and GSE74964, respectively ${ }^{33}$. Mouse brain AUTS2, Bmil, and RING1B ChIP-seq data were obtained from GSE60409 ${ }^{30}$. The human loci related to depression, SCZ, and ASD were converted to their mouse orthologs and overlapped with loci bearing dynamic $6 \mathrm{~mA}$ changes upon stress. All SRA files were downloaded, converted to FASTQ files and processed as described in the bioinformatics methods section.

Data availability. The sequencing data have been deposited at Gene Expression Omnibus under accession number GSE79543. Other data showing the significant loss- and gain-of-6mA; stress-induced transposon expression; stress-induced gene expression alteration and upregulated genes bearing significant loss-of- $6 \mathrm{~mA}$ are shown in Supplementary Data 1-5. All other relevant data are available from the corresponding author.

Received: 12 April 2017 Accepted: 25 August 2017 Published online: 24 October 2017

\section{References}

1. Ma, D. K. et al. Epigenetic choreographers of neurogenesis in the adult mammalian brain. Nat. Neurosci. 13, 1338-1344 (2010).

2. Yao, B. et al. Epigenetic mechanisms in neurogenesis. Nat. Rev. Neurosci. 17, 537-549 (2016).

3. Wion, D. \& Casadesus, J. N6-methyl-adenine: an epigenetic signal for DNAprotein interactions. Nat. Rev. Microbiol. 4, 183-192 (2006).

4. Fu, Y. et al. N6-methyldeoxyadenosine marks active transcription start sites in Chlamydomonas. Cell 161, 879-892 (2015).

5. Greer, E. L. et al. DNA methylation on N6-adenine in C. elegans. Cell 161, 868-878 (2015)

6. Zhang, G. et al. N6-methyladenine DNA modification in Drosophila. Cell 161, 893-906 (2015)

7. Koziol, M. J. et al. Identification of methylated deoxyadenosines in vertebrates reveals diversity in DNA modifications. Nat. Struct. Mol. Biol. 23, 24-30 (2016).

8. Liu, J. et al. Abundant DNA 6mA methylation during early embryogenesis of zebrafish and pig. Nat. Commun. 7, 13052 (2016).

9. Wu, T. P. et al. DNA methylation on $\mathrm{N}(6)$-adenine in mammalian embryonic stem cells. Nature 532, 329-333 (2016).

10. Luo, G. Z., Blanco, M. A., Greer, E. L., He, C. \& Shi, Y. DNA N(6)methyladenine: a new epigenetic mark in eukaryotes? Nat. Rev. Mol. Cell Biol. 16, 705-710 (2015)

11. Sullivan, P. F., Neale, M. C. \& Kendler, K. S. Genetic epidemiology of major depression: review and meta-analysis. Am. J. Psychiatry 157, 1552-1562 (2000)

12. Caspi, A. et al. Influence of life stress on depression: moderation by a polymorphism in the 5-HTT gene. Science 301, 386-389 (2003).

13. Risch, N. et al. Interaction between the serotonin transporter gene (5HTTLPR), stressful life events, and risk of depression: a meta-analysis. J. Am. Med. Assoc. 301, 2462-2471 (2009)

14. Menke, A. \& Binder, E. B. Epigenetic alterations in depression and antidepressant treatment. Dialogues Clin. Neurosci. 16, 395-404 (2014).

15. Sun, H., Kennedy, P. J. \& Nestler, E. J. Epigenetics of the depressed brain: role of histone acetylation and methylation. Neuropsychopharmacology 38, 124-137 (2013).

16. Cerqueira, J. J., Mailliet, F., Almeida, O. F. X., Jay, T. M. \& Sousa, N. The prefrontal cortex as a key target of the maladaptive response to stress. $J$. Neurosci. 27, 2781-2787 (2007).

17. Radley, J. J. et al. Repeated stress induces dendritic spine loss in the rat medial prefrontal cortex. Cereb. Cortex 16, 313-320 (2006).

18. Elliott, E. et al. Dnmt3a in the medial prefrontal cortex regulates anxiety-like behavior in adult mice. J. Neurosci. 36, 730-740 (2016).

19. O’Mahony, C. M., Sweeney, F. F., Daly, E., Dinan, T. G. \& Cryan, J. F. Restraint stress-induced brain activation patterns in two strains of mice differing in their anxiety behaviour. Behav. Brain Res. 213, 148-154 (2010).

20. Zhang, G. et al. Combined use of spatial restraint stress and middle cerebral artery occlusion is a novel model of post-stroke depression in mice. Sci. Rep. $\mathbf{5}$, 16751 (2015)

21. Hains, A. B. \& Arnsten, A. F. Molecular mechanisms of stress-induced prefrontal cortical impairment: implications for mental illness. Learn. Mem. 15 551-564 (2008).

22. Arnsten, A. F. Stress signalling pathways that impair prefrontal cortex structure and function. Nat. Rev. Neurosci. 10, 410-422 (2009) 
23. Robinson, M. D., McCarthy, D. J. \& Smyth, G. K. edgeR: a Bioconductor package for differential expression analysis of digital gene expression data. Bioinformatics 26, 139-140 (2010).

24. Hunter, R. G., McEwen, B. S. \& Pfaff, D. W. Environmental stress and transposon transcription in the mammalian brain. Mob. Genet. Elem. 3, e24555 (2013).

25. Luo, G. Z. et al. Characterization of eukaryotic DNA N(6)-methyladenine by a highly sensitive restriction enzyme-assisted sequencing. Nat. Commun. 7, 11301 (2016).

26. Love, M. I., Huber, W. \& Anders, S. Moderated estimation of fold change and dispersion for RNA-seq data with DESeq2. Genome Biol. 15, 550 (2014).

27. Sannino, G. et al. Acute stress enhances the expression of neuroprotection- and neurogenesis-associated genes in the hippocampus of a mouse restraint model. Oncotarget 7, 8455-8465 (2016).

28. McEwen, B. S. et al. Mechanisms of stress in the brain. Nat. Neurosci. 18, 1353-1363 (2015).

29. Shen, Y. et al. A map of the cis-regulatory sequences in the mouse genome. Nature 488, 116-120 (2012)

30. Gao, Z. et al. An AUTS2-Polycomb complex activates gene expression in the CNS. Nature 516, 349-354 (2014).

31. Mo, A. et al. Epigenomic signatures of neuronal diversity in the mammalian brain. Neuron 86, 1369-1384 (2015).

32. Guo, J. U. et al. Distribution, recognition and regulation of non-CpG methylation in the adult mammalian brain. Nat. Neurosci. 17, 215-222 (2014).

33. Halder, R. et al. DNA methylation changes in plasticity genes accompany the formation and maintenance of memory. Nat. Neurosci. 19, 102-110 (2016).

34. McLaughlin, R. N. Jr. et al. Positive selection and multiple losses of the LINE-1derived L1TD1 gene in mammals suggest a dual role in genome defense and pluripotency. PLoS Genet. 10, e1004531 (2014).

35. Abboud, N. et al. A cohesin-OCT4 complex mediates Sox enhancers to prime an early embryonic lineage. Nat. Commun. 6, 6749 (2015).

36. Hyde, C. L. et al. Identification of 15 genetic loci associated with risk of major depression in individuals of European descent. Nat. Genet. 48, 1031-1036 (2016).

37. Basu, S. N., Kollu, R. \& Banerjee-Basu, S. AutDB: a gene reference resource for autism research. Nucleic Acids Res. 37, D832-D836 (2009).

38. Jia, P., Sun, J., Guo, A. Y. \& Zhao, Z. SZGR: a comprehensive schizophrenia gene resource. Mol. Psychiatry 15, 453-462 (2010).

39. Flint, J. \& Eskin, E. Genome-wide association studies in mice. Nat. Rev. Genet. 13, 807-817 (2012).

40. Uchida, S. et al. Epigenetic status of Gdnf in the ventral striatum determines susceptibility and adaptation to daily stressful events. Neuron 69, 359-372 (2011).

41. Murgatroyd, C. et al. Dynamic DNA methylation programs persistent adverse effects of early-life stress. Nat. Neurosci. 12, 1559-1566 (2009).

42. Haghighi, F. et al. Increased DNA methylation in the suicide brain. Dialogues Clin. Neurosci. 16, 430-438 (2014).

43. Lichtsteiner, S. \& Schibler, U. A glycosylated liver-specific transcription factor stimulates transcription of the albumin gene. Cell 57, 1179-1187 (1989).

44. Porsolt, R. D., Le Pichon, M. \& Jalfre, M. Depression: a new animal model sensitive to antidepressant treatments. Nature 266, 730-732 (1977).

45. Steru, L., Chermat, R., Thierry, B. \& Simon, P. The tail suspension test: a new method for screening antidepressants in mice. Psychopharmacology 85, 367-370 (1985).

46. Song, C. X. et al. Selective chemical labeling reveals the genome-wide distribution of 5-hydroxymethylcytosine. Nat. Biotechnol. 29, 68-72 (2011).

47. Schneider, C. A., Rasband, W. S. \& Eliceiri, K. W. NIH Image to ImageJ: 25 years of image analysis. Nat. Methods 9, 671-675 (2012).

48. Langmead, B., Trapnell, C., Pop, M. \& Salzberg, S. L. Ultrafast and memoryefficient alignment of short DNA sequences to the human genome. Genome Biol. 10, R25 (2009).
49. Shen, L., Shao, N., Liu, X. \& Nestler, E. ngs.plot: quick mining and visualization of next-generation sequencing data by integrating genomic databases. $B M C$ Genom. 15, 284 (2014).

50. Heinz, S. et al. Simple combinations of lineage-determining transcription factors prime cis-regulatory elements required for macrophage and B cell identities. Mol. Cell 38, 576-589 (2010).

51. Medina-Rivera, A. et al. RSAT 2015: regulatory sequence analysis tools. Nucleic Acids Res. 43, W50-W56 (2015).

52. Trapnell, C. et al. Differential gene and transcript expression analysis of RNAseq experiments with TopHat and Cufflinks. Nat. Protoc. 7, 562-578 (2012).

53. Ashburner, M. et al. Gene ontology: tool for the unification of biology. The gene ontology consortium. Nat. Genet. 25, 25-29 (2000).

54. Jin, Y., Tam, O. H., Paniagua, E. \& Hammell, M. TEtranscripts: a package for including transposable elements in differential expression analysis of RNA-seq datasets. Bioinformatics 31, 3593-3599 (2015).

55. Walter, W., Sanchez-Cabo, F. \& Ricote, M. GOplot: an R package for visually combining expression data with functional analysis. Bioinformatics 31, 2912-2914 (2015).

56. Ramírez, F., Dündar, F., Diehl, S., Grüning, B. A. \& Manke, T. deepTools: a flexible platform for exploring deep-sequencing data. Nucleic Acids Res. 42, W187-W191 (2014).

\section{Acknowledgements}

We would like to thank B.L. Philips for critical reading of the manuscript. This work was supported in part by NIH grants (NS051630, NS079625, MH102690 and NS097206 to P.J.)

\section{Author contributions}

B.Y., Y.C. and P.J. conceived and designed the project. B.Y., Y.C., Z.W., Y.L., L.H., W.Z. and D.C. performed the experiments. B.Y. performed the bioinformatics analyses. L.C. assisted with the computational coding. D.C. and B.T. contributed the reagents. B.Y. and P.J. wrote the manuscript. All authors commented on the manuscript.

\section{Additional information}

Supplementary Information accompanies this paper at doi:10.1038/s41467-017-01195-y.

Competing interests: The authors declare no competing financial interests.

Reprints and permission information is available online at http://npg.nature.com/ reprintsandpermissions/

Publisher's note: Springer Nature remains neutral with regard to jurisdictional claims in published maps and institutional affiliations.

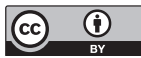

Open Access This article is licensed under a Creative Commons Attribution 4.0 International License, which permits use, sharing, adaptation, distribution and reproduction in any medium or format, as long as you give appropriate credit to the original author(s) and the source, provide a link to the Creative Commons license, and indicate if changes were made. The images or other third party material in this article are included in the article's Creative Commons license, unles indicated otherwise in a credit line to the material. If material is not included in the article's Creative Commons license and your intended use is not permitted by statutory regulation or exceeds the permitted use, you will need to obtain permission directly from the copyright holder. To view a copy of this license, visit http://creativecommons.org/ licenses/by/4.0/.

(c) The Author(s) 2017 